\title{
Challenges and Perspectives in the Macromolecular Flatland
}

\author{
Marco Servalli*
}

\begin{abstract}
Polymer chemistry has recently welcomed a new addition to its field: the planar macromolecules known as two-dimensional polymers (2DPs). These topologically planar and crystalline monolayer covalent sheets are reminiscent of molecular fishermen's nets and apart from being conceptually very interesting for the field of macromolecular chemistry, they also show some potential applications as novel 2D materials. This article reviews how the field has developed five years after the first 2DP was synthesised in 2012. After a brief historical introduction, the main synthetic approaches will be discussed providing concrete examples of 2DPs and highlighting the challenges associated with the synthesis and especially structural characterisation of these fascinating macromolecules. Finally an overview on their potential applications such as membranes for gas separation, rewritable molecular paper and miniaturisation of optical devices will be presented.
\end{abstract}

Keywords: Air/water interface · Crystalline monolayers · Molecular sheets · Topochemical reactions · Twodimensional polymers

\section{Introduction}

Approximately 100 years ago, in 1920, Hermann Staudinger published a paper in which he demonstrated the existence of what at the time was deemed unrealistic: giant molecules with molecular weights easily exceeding $5000 \mathrm{Da}$, which he termed macromolecules (Makromoleküle) or polymers. ${ }^{[1,2]}$ Polymeric materials, such as vulcanised rubber and a variety of nitrocellulose derivatives were already known and being commercialised by the beginning of the 20th century; ${ }^{[3]}$ their molecular structure and the chemistry involved in their preparation were however unknown, and the polymers produced were basically the result of trial-and-error experiments. ${ }^{[4]}$ The unusual properties of polymers were believed to be caused by the aggregation of small molecules, held together by weak intermolecular forces, until Staudinger came up with the revolutionary concept that polymers instead consisted of long linear molecular chains with very high molecular weights, composed of a large number of small molecules linked together by strong covalent bonds. This understanding of the molecular structure of polymers transformed what was once mockingly referred

\footnotetext{
${ }^{*}$ Correspondence: Dr. M. Servalli Laboratory of Polymer Chemistry Department of Materials ETH Zurich, $\mathrm{CH}-8093$ Zurich

E-mail: marco.servalli@mat.ethz.ch
}

to as grease chemistry (Schmierenchemie) into a standalone branch of chemistry and paved the way to synthetic polymers, which literally conquered the global markets, with a current yearly production in the order of 311 million tonnes. ${ }^{[5]}$ The success of polymers lies in their versatility and easy accessibility provided by the vast arsenal of organic chemical reactions that can be employed for their production: by starting from the synthesis of the monomers themselves, which can be equipped with defined chemical functionalities, by covalently linking them together through controlled and selective polymerisation reactions and finishing with post-polymerisation modifications, polymers can be tailor-made having the desired properties according to societal needs.

Before venturing into the main topic of this article, let's have a more detailed look at the aforementioned linear macromolecules; is there a common characteristic among the huge variety of polymers being produced nowadays? What does polyethylene used in plastic bags have in common with, for instance, polyamide-imides found in airplane parts? The answer lies in the topology of their polymerisation reactions. During the growth reaction, the monomers are covalently attached together following a hypothetical line, resulting in a chain of repeat units with one-dimensional topology. Coiling of the chains and entanglements do not compromise their topological 1D character; the same holds for branching or cross-linking, as the chains growing from the branching points or the ones that are cross-linked are always grown one-dimensionally. In this sense, the synthetic polymers have not only conquered the global markets, but also the first dimen- sion from a topological point of view. The spontaneous question that now arises is, if it is also possible to have polymers that are topologically two-dimensional, namely having a growth reaction in which the monomers are linked together on a plane rather than a line, forming topologically planar sheets of repeat units.

The answer is provided by graphene, which is in fact a natural two-dimensional polymer (2DP). Its periodical structure is composed of carbon atoms only, which are linked together by strong covalent bonds and ensure the atom-thick sheet to be free-standing. Staudinger's concept of polymers composed by repeat units can be applied to graphene as well, by considering, for instance, a single $\mathrm{sp}^{2}$-hybridised carbon as repeat unit; in this case, however, the repeat units have three laterally extended bonded sites and are connected together on a plane rather than a line (Fig. 1), resulting in a topologically planar sheet. The outstanding properties of graphene are due to its peculiar structure and particularly its confinement into two dimensions and since its isolation and characterisation in $2004,[6,7]$ the interest and research for other monolayer 2D materials has increased dramatically; a few examples include other elemental allotropes such as silicene, ${ }^{[8,9]}$ germanene, ${ }^{[10]}$ stanene. ${ }^{[11,12]}$ and phosphorene, ${ }^{[13]}$ hexagonal boron nitride, ${ }^{[14,15]}$ and a variety of inorganic metal dichalcogenides such as $\mathrm{MoS}_{2}, \mathrm{WS}_{2}, \mathrm{TaS}_{2}, \mathrm{MoSe}_{2}$ and $\mathrm{NbSe}_{2}{ }^{[16,17]}$ The methods developed for accessing these materials as monolayers can vary: top-down approaches such as micromechanical or liquid exfoliation ${ }^{[17-20]}$ can be employed when 2D materials exist in layered form such as graphene in graphite, phosphorene in black phos- 
a) 1D polymer - Repeat units topologically connected on a line

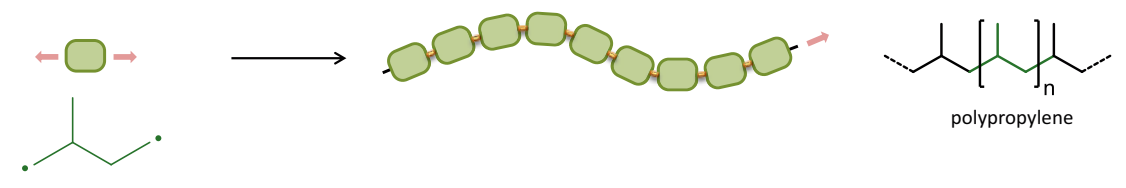

b) $2 \mathrm{D}$ polymer - Repeat units topologically connected on a plane
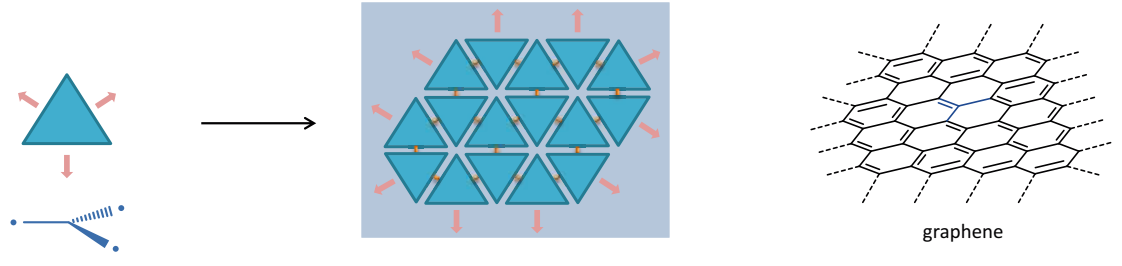

Fig. 1. Cartoon representation of the topological differences between 1D and 2D polymers. a) In linear polymers, monomers have two binding sites and during the growth reaction they get connected to each other along a hypothetical line, resulting in a chain-like entity composed of repeat units that extend topologically in one dimension, such in the case of polypropylene (repeat unit 1,2-propandiyl marked in green); b) In 2D polymers instead, monomers have typically three binding sites arranged in a planar geometry which get connected together during the growth reaction on a hypothetical plane, resulting in a sheet-like entity with repeat units extending topologically in 2D, such as in the case of graphene, where the repeat unit can be considered as a single $\mathrm{sp}^{2}$-hybridised carbon atom.

phorous, or the various transition metal dichalcogenides; alternatively, bottom-up approaches have also been developed such as chemical vapour deposition (CVD) ${ }^{[21-23]}$ and thermolysis, ${ }^{[24,25]}$ which however require very harsh conditions, unthinkable for standard organic chemistry.

In fact, while there is no doubt about their interesting properties and accessibility, the aforementioned 2D materials are mostly of inorganic nature, and from the eye of a synthetic chemist, the lack of functional groups in their structures can appear as a great limitation in terms of their potential use, even though some of them can be functionalised to some extent, such as graphene, ${ }^{[26-28]}$ hexagonal boron nitride ${ }^{[29]}$ and silicene. ${ }^{[30]}$ After all, the success of linear polymers is due to their chemical versatility and tuneable properties. It therefore appears very attractive to access $2 \mathrm{D}$ materials the same way synthetic 1D polymers were created, namely by designing monomers with the desired functionalities, and polymerise them two-dimensionally by using the mild recipes that organic chemistry can offer, creating synthetic 2DPs.

Attempts to confine polymerisations in $2 \mathrm{D}$ are not new and the first experiments with monolayers at the air/water interface date back to $1935 ;{ }^{[31,32]}$ other attempts followed ${ }^{[33-36]}$ but they relied on linear polymerisations to create $2 \mathrm{D}$ molecular objects rather than employing real 2D growth reactions. The breakthrough finally came in 2012, when the first synthetic 2DP was created and characterised by our group, ${ }^{[37]}$ showing that it is indeed possible to make two-dimensional sheet-like macromolecules composed of repeat units. Let's therefore venture into the macromolecular flatland and see how polymers are also slowly conquering the second dimension, starting by the definition of $2 \mathrm{DP}$.

\section{Definition of 2DP}

Five defining criteria were proposed: 1) Planarity: a $2 \mathrm{DP}$ is a topologically planar molecular sheet;

2) Periodicity: a 2DP has a periodically ordered structure and exhibits crystallinity in at least one of its conformations. Periodicity is conferred by the repeat units in its structure;

3) Robust bonds: the repeat units in a 2DP are linked together by robust bonds, preferably of covalent nature;

4) Thickness: a 2DP is a monolayer whose thickness corresponds to the thickness of its constitutional repeat unit;

5) Free-standing: the bonds between the repeat units are robust enough to ensure that a 2DP is free-standing and can be manipulated in its monolayer form.

This definition was proposed by the Schlüter group ${ }^{[36]}$ and is increasingly becoming accepted. Other views on the definition of 2DPs exist ${ }^{[38-40]}$ which also encompass multilayers and non-periodical structures. However the concept of a repeat unit is at the very core of the definition of a polymer and in our view it cannot be neglected: as a matter of fact some properties of 2DPs are expected to manifest due to their structural periodicity and monolayer nature. As such, molecular entities which do not fulfil all five criteria can be best addressed with the more general term $2 \mathrm{D}$ materials.

\section{Approaches to Synthetic 2DPs}

Synthesising a 2DP is not an easy task: first of all, one starts with the monomer, whose design has to be carefully thought in terms of connection chemistry and according to the method intended to be used for the synthesis of a 2DP. As a general design criterion, in order to span a periodical two-dimensional network, the monomer should have three-, four- or six-fold symmetry. For the polymerisation step, the biggest challenge lies in having a controlled growth reaction confined in $2 \mathrm{D}$ and avoiding its departure into the third dimension with consequent formation of a 3D network. This point can be addressed by pre-organising the monomers in two dimensions before triggering the polymerisation reaction. The two most successful synthetic methods in this regard rely on the pre-organisation of the monomers into layered single crystals and at the air/water interface. These two methods will be described in the following paragraphs, highlighting their weaknesses and strengths and giving concrete examples of synthesised 2DPs. Alternative and promising synthetic methods will also be addressed and briefly discussed at the end of this section.

\subsection{Topochemical Single Crystal Approach}

The first synthetic method is based on the topochemical polymerisation of monomers in single crystals. Topochemistry was developed by Schmidt and Cohen in the 60 s and $70 \mathrm{~s}^{[41,42]}$ and was already exploited by Wegner and Hasegawa to create linear polymers from difunctional molecules such as diacetylenes ${ }^{[43]}$ and diololefins ${ }^{[44]}$ via photopolymerisations in crystals.

The topochemical approach for the synthesis of 2DPs involves the crystallisation of a monomer into lamellar single crystals (Fig. 2). In each layer, the monomers are regularly packed with their reacting sites in close proximity to their neighbours. The layers are held together by weak intermolecular forces and ideally do not have the chance to chemically cross-link with each other: the directionality of the bonding sites of the monomers is 2D-confined in each layer, so that the growth reaction will also be confined in two dimensions. Thermal treatment or photoirradiation of the crystal then triggers the polymerisation reaction, with the formation of covalent bonds between the monomers, converting the monomer crystal into a $2 \mathrm{D}$ polymer crys- 


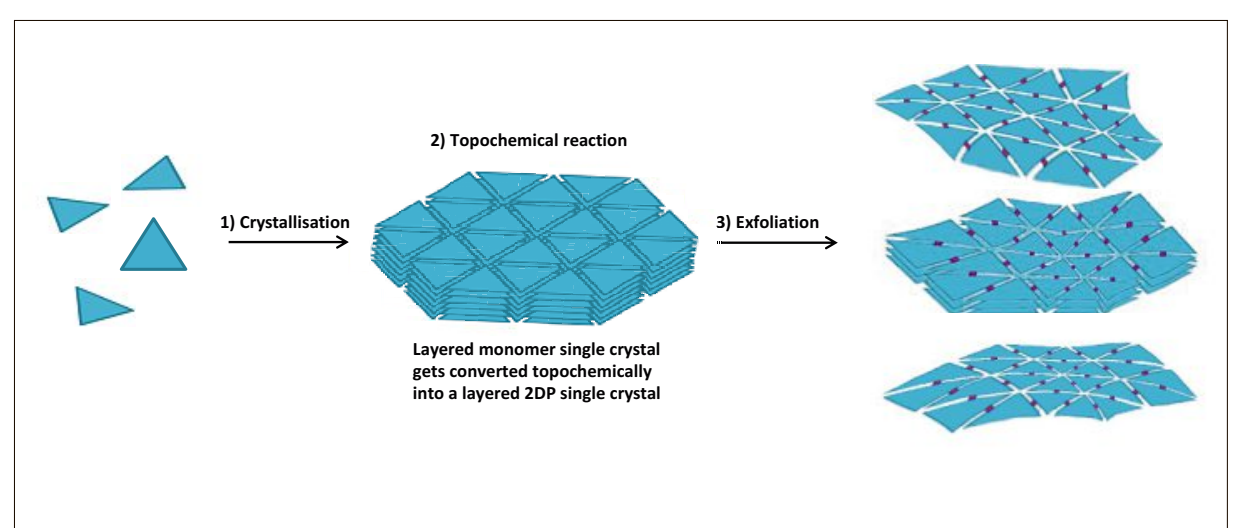

Fig. 2. Cartoon representation of the topochemical single crystal approach for the synthesis of 2DPs. 1) The monomers are crystallised into layered single crystals, in which the reactive sites of the monomers are in close proximity to each other; 2) A topochemical reaction converts the layered monomer crystal into a 2DP crystal; 3) Exfoliation of the layered 2DP crystals results in thin sheet stacks and eventually in single layers.

tal. Thermal and photo-induced reactions such as cycloadditions are the most attractive choices since they do not involve any mass transport associated with common reagents. 2DPs synthesised by this method so far involved photoinduced [4+4]-cycloadditions between face-to-face stacked anthracene units, [4+2]-cycloadditions between anthracene and acetylenic units and $[2+2]$-cycloadditions between olefins. It is important to note that these kinds of solid-state reactions are always associated with changes in the lattice parameters of mother and product crystals, caused by the small rearrangements of the molecules during the reaction due to the conversion of van der Waals interactions (with distances typically between 3.5 and $4.2 \AA$ ) into short covalent bonds. Consequently two possible scenarios can occur: a) molecular movement in the crystal during the reaction is minimal, reflecting minimal changes in the lattice parameters between monomer and polymer crystals and resulting in a single-crystal-to-single-crystal (SCSC) transformation. b) molecular rearrangements are important and lattice parameters vary considerably during the reaction, resulting in important mechanical strains in the crystal; in this case the crystal might crack or break (with the pieces still being single crystalline), lose crystallinity or in the worst case disintegrate into a powder. Scenario a) is clearly more attractive, as the polymer crystal can be easily characterised by single crystal X-ray diffraction (SC-XRD), giving unequivocal proof of the structure of the 2DP; in scenario b), even if the reaction might proceed topochemically, the loss of crystallinity in the product crystal can prevent the use of $\mathrm{SC}-\mathrm{XRD}$, rendering the characterisation of the 2DP less trivial.

As last step of the single crystal approach, the $2 \mathrm{D}$ polymer crystal can then be subjected to a process of solvent-mediated wet exfoliation in order to obtain thin sheet packages and eventually single layers.

The five examples of 2DPs synthesised so far by this method are summarised in Fig. 3 and will be discussed in the next paragraphs. The monomer structures are displayed with their reactive units indicated in red colour, together with the connection chemistry employed for polymerisation and the structures of the obtained 2DPs. For monomers $\mathbf{1}$ and $\mathbf{2}$, which polymerise topochemically, crystal structures of the obtained polymers are not available, whereas for monomers $\mathbf{3}, \mathbf{4}$ and $\mathbf{5}$, crystal structures of monomer and polymers are provided, showing the SCSC nature of these polymerisations.

The prototype organic 2DP was prepared in 2012 with this method in our group by Kissel et al., [37] by synthesising the anthracene-based, cup-shaped rigid macrocycle 1 displayed in Fig. 3a. The monomer was crystallised, and the obtained single crystals were analysed by $\mathrm{SC}-\mathrm{XRD}$, revealing a layered structure in which the anthracene units of the monomers were arranged in close proximity with the acetylenic moieties of their neighbours. Despite violating the orbital symmetry rules, ${ }^{[45]}$ photoirradiation triggered a [4+2]-cycloaddition reaction polymerising the monomers in the layers. The reaction proceeded topochemically but according to the scenario b) discussed previously: the loss of crystallinity in the polymerised crystal prevented analysis by SC-XRD. Bond formation was therefore confirmed by Raman and solid-state NMR spectroscopy, and structural periodicity by electron diffraction. Wet exfoliation of the polymerised crystals resulted in monolayers, whose thickness was confirmed by atomic force microscopy (AFM).

This case confirmed the potential of the topochemical single crystal approach for the synthesis of 2DPs and one year later, in 2013 the trifunctional anthracene-based monomer 'antrip' 2 developed by the King group, also resulted in a 2DP as described in the work of Bhola et al.:[46] the monomer was crystallised, revealing a pseudo-lamellar structure (Fig. 3b); the proximity and alignment of the anthracene units of the monomers was however sufficient for polymerisation to occur via photoinduced [4+4]-cycloaddition. The reaction proceeded topochemically but with loss of crystallinity in the product crystals, preventing SC-XRD analysis; bond formation was therefore confirmed by IR- and solid-state NMR spectroscopy and the crystals could be wet-exfoliated into single sheets as confirmed by AFM.

The first crystal structures of two 2DPs were reported one year later in two independent works from the King group and our group. In the first work from Kissel et al., ${ }^{[47]}$ a fluorinated version of the antrip monomer, the 'fantrip' 3 was crystallised and polymerised in two consecutive steps by the photoinduced [4+4]-cycloaddition reaction between the fluorinated anthracene units (Fig. 3c). The reaction occurred in a SCSC fashion according to the scenario a) described previously so that dimer and polymer crystal structures could be obtained by SC-XRD. The 2DP single crystals could be wet-exfoliated yielding thin sheet stacks and also monolayer sheets.

The second work was provided by the trifunctional anthracene-based monomer 4 made by Kory et al. ${ }^{[48]}$ (Fig. 3d). The monomer was crystallised and polymerised via [4+4]-cycloaddition of its anthracene units. The reaction proceeded again in a SCSC fashion and the structure of the 2DP was characterised by SC-XRD; reversibility of the polymerisation reaction was also demonstrated by a thermally-induced depolymerisation at $180{ }^{\circ} \mathrm{C}$, which also proceeded in a SCSC fashion, demonstrating the ability of the single crystals to switch between polymerised and depolymerised state without losing crystallinity. In this case however, the exfoliation procedure was challenging due to the very strong interactions between the polymer layers in the crystals, yielding mostly stacks of 10-50 sheets and only rarely monolayers. Compared to the previous examples, this monomer can be synthesised on a multigram scale, accessing the corresponding 2DP crystals in quantities interesting for technical applications.

The latest case of 2DP crystal structure is based on the trifunctional monomer bearing styryl pyrylium moieties $\mathbf{5}^{\text {[49] }}$ (Fig. $3 \mathrm{e})$. This easily accessible ionic compound crystallises in layers and undergoes $2 \mathrm{D}$ polymerisation via photochemically induced $[2+2]$-cycloaddition between the double bonds of its styryl units. ${ }^{[50]}$ The reaction proceeds in a SCSC fashion yielding 2DP 


\section{Monomer}

a)

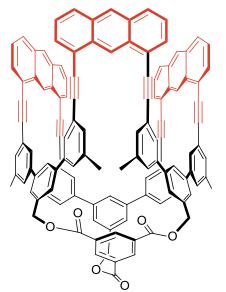

1

b)

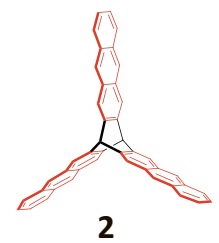

c)

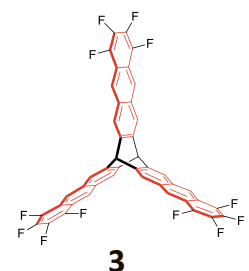

3

d)

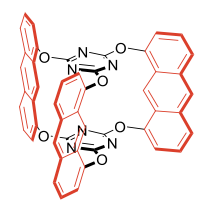

4

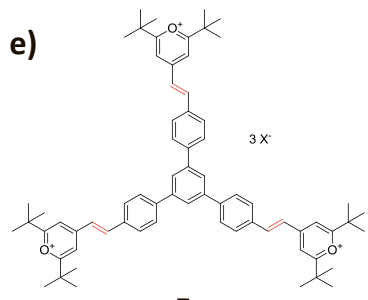

5
Connection chemistry

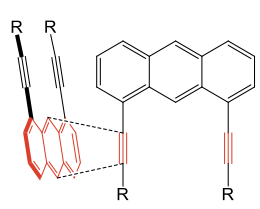

Photoinduced [4+2]-cycloaddition between anthracene and triple bond. Topochemical reaction
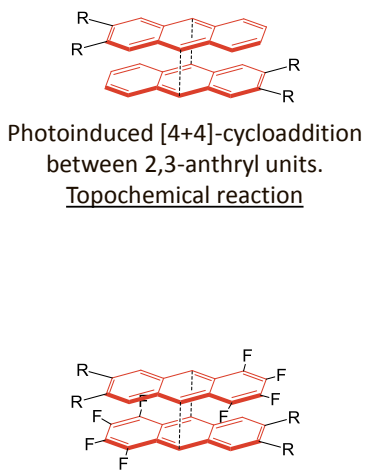

Photoinduced [4+4]-cycloaddition between fluorinated 2,3-anthryl units. Topochemical SCSC reaction
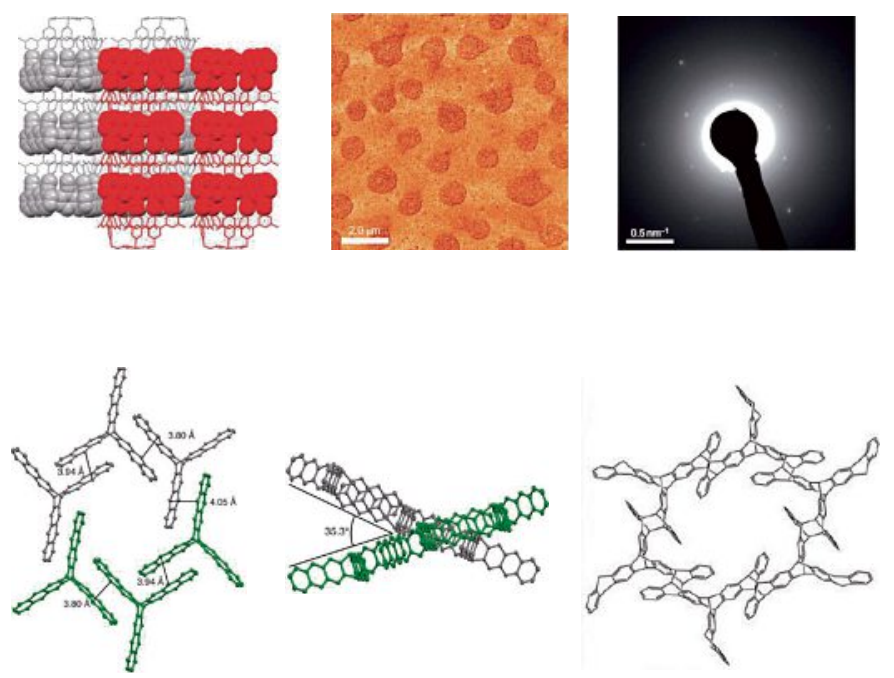

1) $400 \mathrm{~nm}$

2) $460 \mathrm{~nm}$

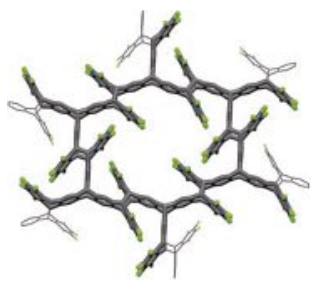

$465 \mathrm{~nm}$

$180^{\circ} \mathrm{C}$

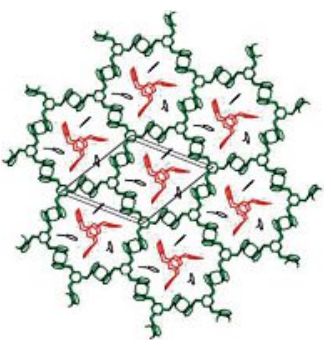

between 1,8-anthryl units.

Topochemical SCSC reaction
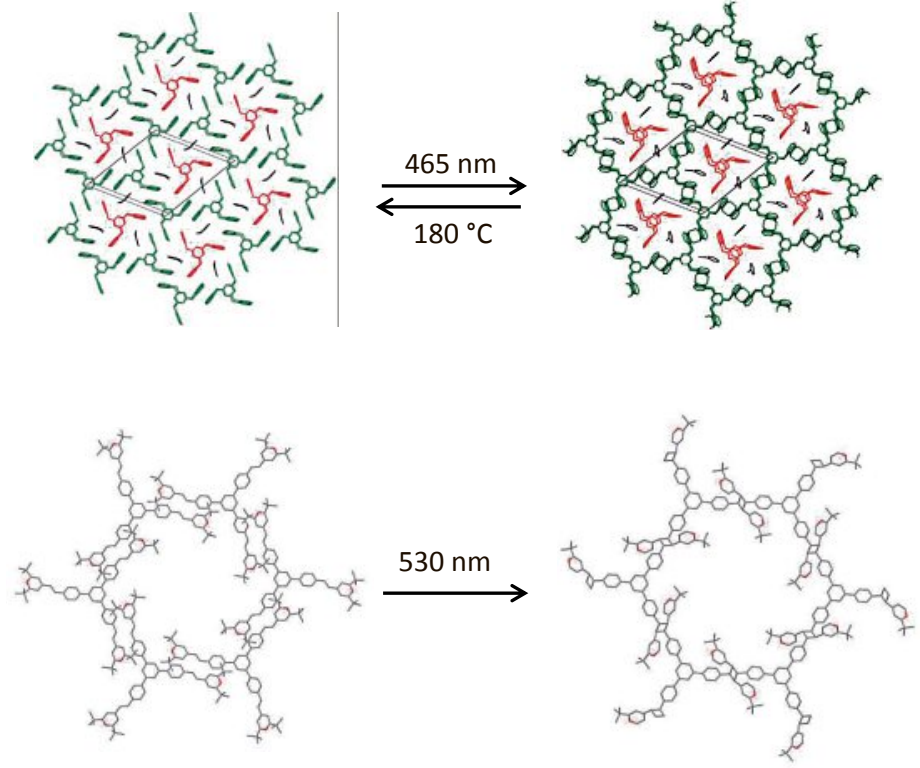

Fig. 3. The five cases of 2DPs synthesised by the single crystal approach. All cases are based on photochemically triggered cycloadditions. In the monomer structures the photoreactive units are marked in red colour. a) The prototype synthetic polymer from Kissel et al.: the cup-shaped monomer 1 was crystallised and polymerised via photoinduced [4+2]-cycloaddition of its anthracene and acetylenic moieties. From left to right: layered crystal structure of the monomer; AFM image of the monolayers obtained from the exfoliation of the polymer crystals; ED pattern of the polymer crystals indicating crystallinity. Adapted with permission from ref. [37]. b) 2DP prepared by Bhola et al.: the antrip monomer 2 was crystallised into pseudo-lamellar single crystals, which polymerised topochemically upon photoirradiation; the proposed structure of the 2D polymer is shown on the right. Adapted with permission from ref. [46]. c) The fluorinated fantrip monomer $\mathbf{3}$ from Kissel et al. polymerised in a two-step topochemical SCSC photopolymerisation. Adapted with permission from ref. [47]. d) The rotor-shaped monomer $\mathbf{4}$ from Kory et al. was photopolymerised and thermally depolymerised via topochemical SCSC reactions. Adapted with permission from ref. [48]. e) The styryl pyrylium salt 5 from Lange et al. was photopolymerised via [2+2]-cycloaddition in a SCSC fashion; the counterions can be either $\mathrm{TfO}^{-}$or $^{\mathrm{BF}_{4}^{-}}$. 
ionic crystals, which can be wet-exfoliated providing a variety of sheet stacks with different thickness and in some cases also elements with monolayer nature.

\subsubsection{Pros and Cons of the Single Crystal Approach}

These five examples clearly show the potential of the single crystal approach for the synthesis of 2DPs. The method can be applied to a variety of different monomeric structures by exploiting photo-induced cycloadditions, and the clear advantage is that a routine technique such as SC-XRD can be applied to characterise the obtained 2DPs, giving unequivocal structural proof. Periodicity in the layers is imparted by nature itself during the crystallisation of the monomers and is transferred in the polymer by topochemistry. However, the approach is associated with challenges at three different levels: 1) Obtaining the desired crystal packing, 2) steering the topochemical reaction towards a SCSC process, 3) exfoliating the layered 2DPs to monolayers. The beauty of the examples exposed previously is that they deal with these three points differently, highlighting the challenges that might be encountered with this method. For instance, as seen in the first two examples of Kissel ${ }^{[37]}$ and Bhola, ${ }^{[46]}$ the passage from monomer to polymer crystal, even if complying with the topochemical principles, does not always proceed in an SCSC fashion. If molecular rearrangement during the polymerisation reaction is important and there is a considerable mismatch in the lattice parameters of monomer and polymer crystals, then the mechanical strains deriving from the process will most likely result in cracks in the crystals, loss of crystallinity, or even complete disintegration of the crystal, preventing the use of SC-XRD analysis on the polymer crystal. In these cases, it is always possible to try and steer the topochemical reaction towards a SCSC transformation by finely tuning the reaction parameters such as temperature, crystal size and shape, wavelength and intensity of the light source. A particularly useful technique developed by Enkelmann concerns the use of tail-irradiation, ${ }^{[51]}$ in which the crystals are irradiated with a wavelength corresponding to the tail-end of their absorption spectrum; this way, the photons are more evenly distributed throughout the entire volume of the crystal, reducing the chances of phase segregations during the irradiation. The strains accumulated in the crystals by the mismatch between lattice parameters of monomer and polymer crystals are dissipated through the crystal surfaces, surfaces in small or thin crystals are more accessible, and the accumulated stress can be released more efficiently, preventing crack formation. ${ }^{[52]}$ Likewise, slow reaction rates are preferred, as the stress in the crystal is accumulated slowly and can be better dissipated: low intensity irradiations should therefore be preferred. The role of the temperature is a bit more complex: high temperatures impart more thermal movement in the crystal, rendering it more 'fluid' and allowing a fast rearrangement of the molecules during the topochemical reaction. This dramatically increases the reaction rate and can result in rapid accumulation of strains. Conversely, low temperatures might hamper the molecular rearrangements necessary for the topochemical reaction to occur. If these expedients do not succeed to retain the single-crystalline nature of the polymer crystals, the characterisation of the 2DP might be less trivial and methods such as IR, Raman and solid-state NMR spectroscopy should be employed to confirm bond formation. Powder XRD and ED analysis can then be employed to confirm the crystallinity of the obtained 2DP.

The next issue associated with this method regards the size of the obtained 2DP sheets; in fact the maximal lateral extension that can be reached by a sheet corresponds to the lateral extension of the single crystal itself. However, single crystals are usually far from ideal and can have defects: incorporation of impurities, dislocations, twinning, grain boundaries are not uncommon and combined with the phenomenon of mosaicity can reduce the size of the periodical domains in a crystal. To tackle this issue, it is important to grow single crystals with the highest quality possible. Growing larger single crystals can yield larger 2DP sheets, but as explained previously, large crystals might not be able to properly dissipate the strains exerted during a topochemical reaction, lowering the chance of a having well-behaved SCSC transformation. Moreover, larger single crystals simply contain more defects that smaller ones.
The exfoliation to single sheets can become challenging when the intermolecular forces between the layers of a 2DP crystal are too strong, as seen in the case of Kory et al. ${ }^{[48]}$ and partially in the case of Lange et al. ${ }^{[49]}$ Moreover, if the exfoliation process is not gentle enough, it could also result in ill-shaped sheets, mostly caused by rupturing or folding.

Last but least, the prerogative for this method to work is to obtain the desired lamellar crystal packing, with the reactive units of the monomers in close proximity able to undergo topochemical polymerisation. This is not a trivial issue, as despite the progress in crystal engineering over the past years, ${ }^{[53]}$ predicting a crystal packing is still a major challenge and the only reliable methods involve the demanding and expensive algorithms of computational structure prediction (CSP). ${ }^{[54,55]}$ Therefore, even by carefully designing the monomer and choosing the proper supramolecular synthons (which in this case mainly imply anthracene derivatives and unsaturated bonds able to undergo cycloadditions), there will always be an element of uncertainty as to whether the monomer will pack in the desired way. For anthracenes to undergo cycloaddition in the solid state for instance, a face-to-face stacked arrangement is essential. The packing problem is accentuated by the phenomenon of polymorphism and that different crystallisation solvents can yield different packings. As a matter of fact, monomer 4 , resulted in a reactive packing suitable for $2 \mathrm{D}$ polymerisation only when crystallised from 2-cyanopyridine, whereas for another monomer, the anthraphane $\mathbf{6},{ }^{[56]}$ a thorough crystallisation study from 30 solvents resulted in a variety of packing motifs (Fig. 4) with different stacking arrangements for the anthracene units, some of which were able to form dimers and 1D polymers, but none of them useful for 2D polymerisation. ${ }^{[57]}$

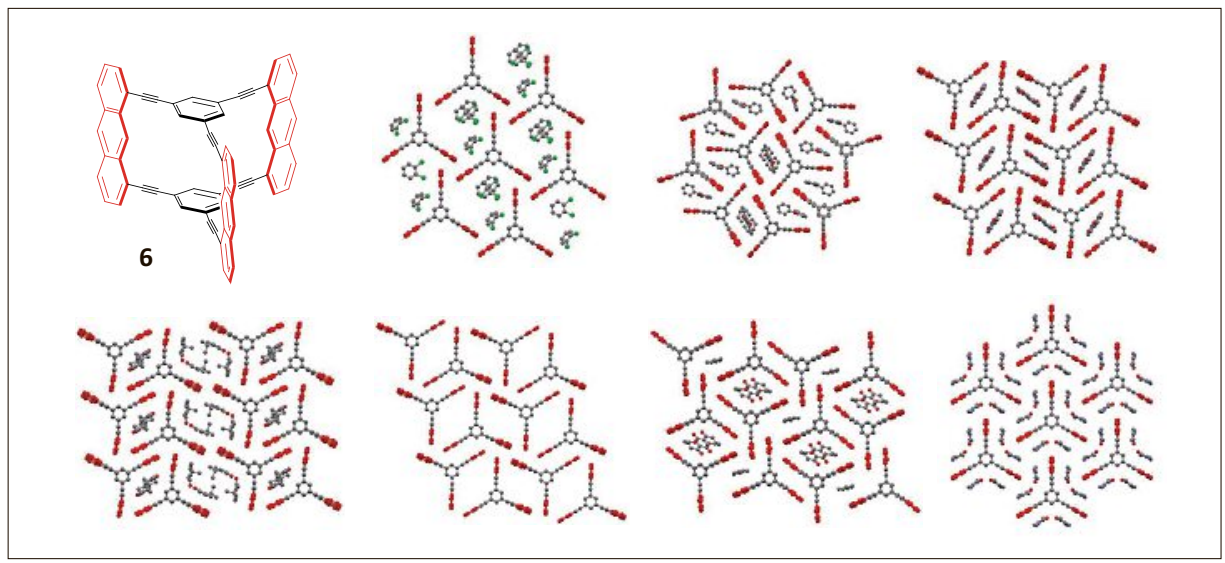

Fig. 4. Anthraphane monomer 6 designed for the single crystal approach. Crystallisation from 30 solvents yielded seven packing motifs, none of which could be used for 2D polymerisation, due to the anthracene units not being entirely face-to-face stacked. 
Despite the potential problems that can be encountered when using the single crystal approach, it has nevertheless proven to be a powerful method for synthesising 2DPs and possibly accessing them in large scale. The challenges regarding crystal packing and exfoliation should not discourage researchers as they can in principle be tackled by a careful monomer design. In this regard, the reported cases of 2DPs and the empirical evidence gathered so far will for sure lead to better and improved designs.

\subsection{Air/Water Interfacial Approach}

The second synthetic method for 2DPs involves the pre-organisation of the monomers at the air/water interface. Pioneering experiments with monolayers at the air/ water interface date back to 1917 with the work of Langmuir with amphiphilic fatty acids, ${ }^{[58,59]}$ while linear polymerisations were first performed by Gee in the 1930s. ${ }^{[31,32]}$ Typically, in this approach amphiphilic monomers with laterally extended binding sites are spread at the air/water interface in a Langmuir trough and are successively mechanically compressed into a monomer monolayer. Upon compression, the monomers pack together, ideally into a crystalline state, with their reactive units in close contact with each other. At this point, an external stimulus such as light irradiation or the diffusion of reagents from the water subphase to the reactive sites triggers the polymerisation (Fig. 5). The monomer monolayer is thus converted directly into a 2DP monolayer. The flatness of the water surface (surface roughness of $3 \AA^{[60]}$ ) ensures that the growth reaction will take place within the monolayer plane. The maximal achievable lateral extension of the monolayers corresponds to the extension of the water surface, thus allowing access to macroscopic sheets.

However, the amount of material spread at the interface is typically in the microgram range, rendering the analysis of the sheets challenging. Characterisation can be performed both in situ at the interface or after transferring the sheets on a substrate. A variety of monolayer-sensitive techniques can be applied to confirm bond formation, such as infra-red reflection absorption spectroscopy (IR-RAS), tip-enhanced Raman spectroscopy (TERS) and X-ray photoelectron spectroscopy (XPS), whereas to analyse the thickness of the polymer, height analysis by atomic force microscopy (AFM) can be performed. The free-standing nature can be confirmed by transferring the monolayer on holey substrates, using scanning electron microscopy (SEM) to see if the film is able to span the holes without collapsing under its own weight. Determining the structural periodicity of the sheets is, however, another

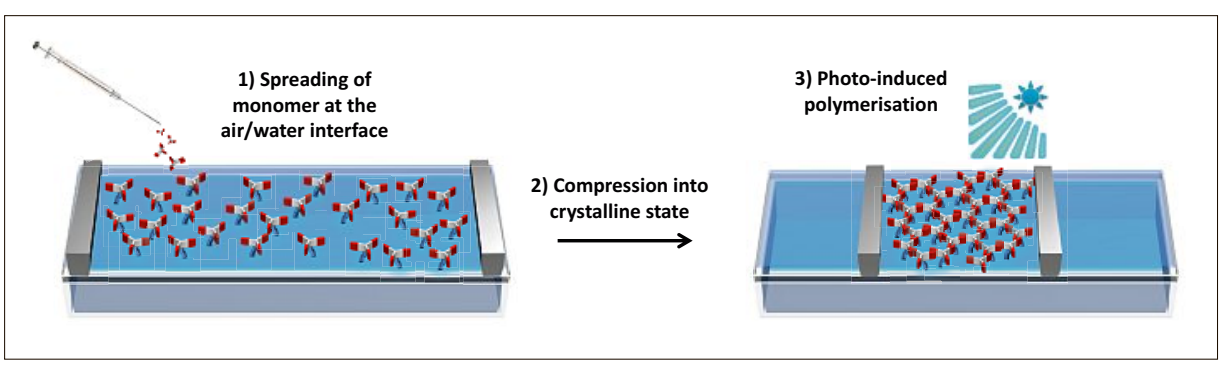

Fig. 5. Cartoon representation of a Langmuir trough employed for the synthesis of 2DPs at the air/ water interface approach.

story as there is no routine technique such as SC-XRD. Analytical methods rely on scanning tunnelling microscopy (STM), high resolution transmission electron microscopy (HR-TEM), high resolution AFM and diffraction techniques such as grazing-incidence small-angle $\mathrm{X}$-ray scattering (GISAXS) and selected area electron diffraction (SAED), all of which require expertise and time. It is therefore common to find in the literature covalent monolayer sheets which still await structural elucidation, whereas sheets that fully meet the five criteria to be classified as 2DPs are still scarce. A few selected examples are summarised in Fig. 6 and will be briefly discussed.

The first examples include photopolymerisation reactions based on irreversible covalent bond formation. Amphiphilic monomer 7a (Fig. 6a) in the work of Payamyar et al..$^{[61]}$ was spread at the air/ water interface and photopolymerised via [4+4]-cycloaddition of its anthracene moieties; bond formation was confirmed by fluorescence spectroscopy and TERS [62] and the resulting covalent monolayer displayed mechanical coherence, however, structural periodicity could not be confirmed. The structural flexibility of the monomer was likely imparted to the polymer upon polymerisation, resulting in a flexible network. This was supported by a DFT simulation that showed how the ideal crystalline structure of the polymer underwent relaxation, collapsing into an amorphous network. Monomers 7b,c also resulted in similar free-standing covalent sheets. [63-65]

The issue of structural flexibility was addressed by the shape-persistent amphiphilic monomer 8 (Fig. 6b) synthesised by the King group in the work of Murray et al. ${ }^{[66]}$ Photopolymerisation produced a covalent monolayer sheet, which could be characterised by STM showing local structural periodicity. This demonstrated the validity of the air/water interface approach for synthesising 2DPs.

The other selected examples concern 2D polymerisations based on coordination chemistry with metal ions, yielding monolayers which can be classified as two-dimensional metal-organic frameworks (2D-MOFs). The hexafunctional terpyridine-based monomer 9 (Fig. 6c) by Bauer et al., ${ }^{[67]}$ was spread at the interface and metal ions such as $\mathrm{Fe}^{2+}$ were supplied to the subphase; diffusion of the cations to the water surface induced complexation between the terpyridine units, connecting the monomers together and resulting in free-standing monolayers. Similar monolayers were obtained with trifunctional terpyridine-based monomers[ ${ }^{[68]}$ and with the trifuntional dipyrrin-based monomer $\mathbf{1 0}$ (Fig. 6d) from the groups of Sakamoto and Nishihara, ${ }^{[69,70]}$ which were complexated by $\mathrm{Zn}^{2+}$ cations. The same groups also employed complexation of benzenehexathiols with $\mathrm{Ni}^{2+}$ ions. ${ }^{[71]}$ Another example of nickel-thiol complexated sheets was provided by the Feng group in the work of Dong et al., using monomer 11 (Fig. 6e) yielding free-standing monolayers. ${ }^{[72]}$ In another example, metallated tetrafunctional porphyrins equipped with carboxylate groups from the Makiura group were complexated at the air/water interface with $\mathrm{Cu}^{2+}$ ions and formation of crystalline monolayer domains could be monitored in situ by grazing incidence $\mathrm{X}$-ray diffraction (GIXRD). [73]

Although there has been progress in analysing the crystallinity of these monolayers by SAED, difficulties associated with Moiré pattern formation make it challenging to provide unequivocal support for the measured values; another important issue is the scarce stability of the monolayers under the electron beam. The latest report in this regard concerns the monomers 12a and $\mathbf{1 2 b}$ (Fig. 6f) in the work of Sahabudeen et al. from the Feng group. ${ }^{[74]}$ The monomers are connected together by condensation reactions forming imine bonds; this kind of dynamic covalent chemistry is interesting as it offers the chance of healing in case of defect formation and produces two-dimensional covalent organic frameworks (2D-COFs). The crystallinity of the obtained monolayer was confirmed locally in different spots by SAED: to enhance the stability of the monolayer towards the electron beam, it was sandwiched between two graphene layers. 
Monomer

a)

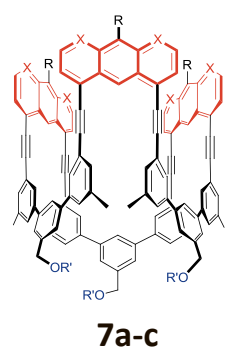

b)

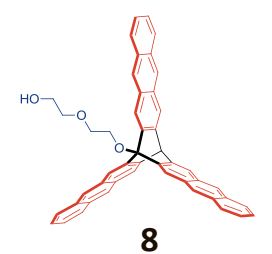

c)

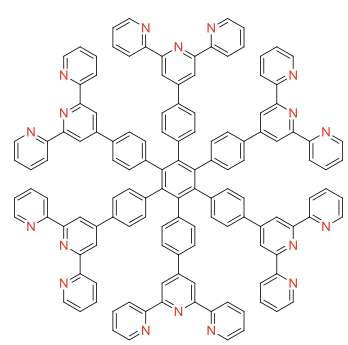

9

d)

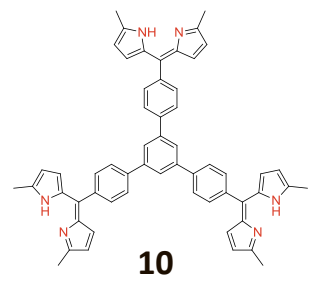

e)

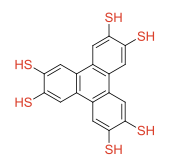

11

f)

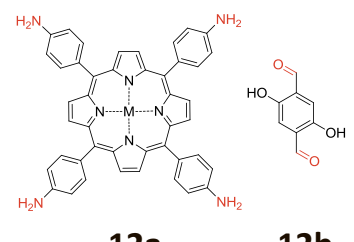

$12 a$
Connection chemistry

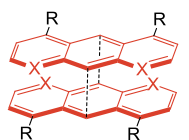

Covalent $\mathrm{C}-\mathrm{C}$ bonds via of 1,8-anthryl units photoinduced [4+4]-cycloaddition

2D polymer
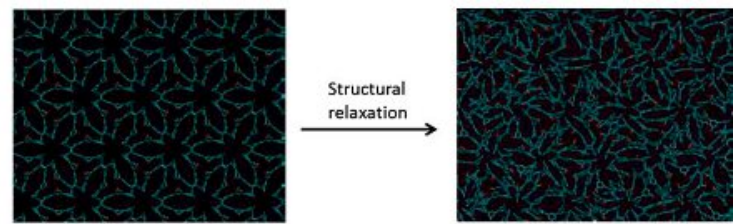

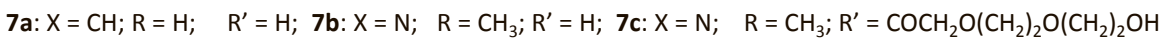

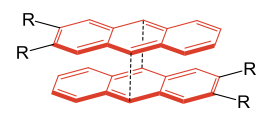

Covalent $\mathrm{C}-\mathrm{C}$ bonds via photoinduced [4+4]-cycloaddition of 2,3-anthryl units

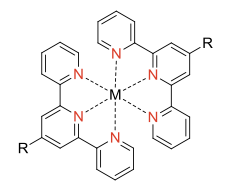

Coordinate bonds between terpyridines units and metal cations such as $\mathrm{Fe}^{2+}$

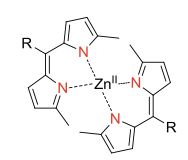

Coordinate bonds between dipyrrol units and $\mathrm{Zn}^{2+}$ cations

$$
\text { 舟 }
$$

Coordinate bonds between thiol units and $\mathrm{Ni}^{2+}$ ions

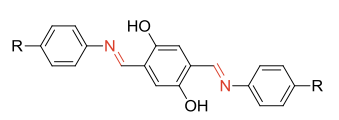

Reversible covalent imine bonds via condensation reaction of amines and aldehydes
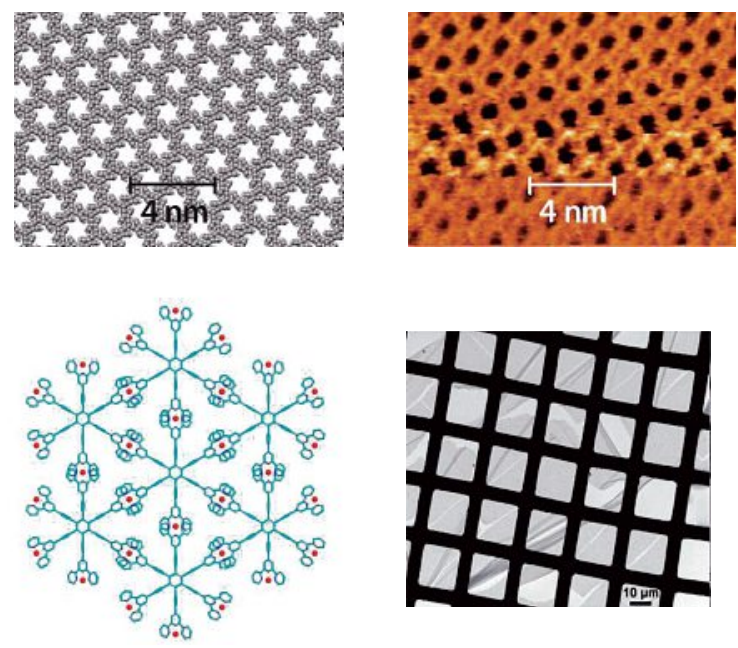
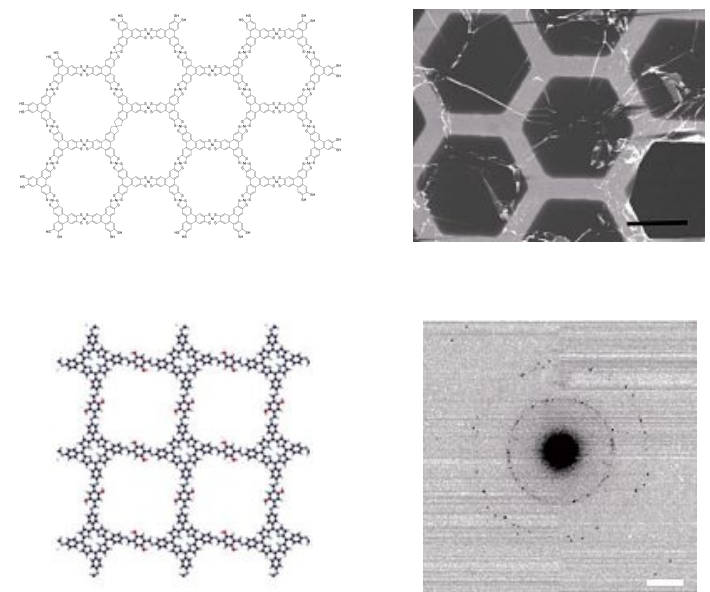

Fig. 6. Selected examples of systems employed for the air/water interface approach. In the monomer structures the reactive units are marked in red colour. a) Cup-shaped amphiphilic monomers 7a-c for synthesis of covalent monolayer sheets; DFT simulation indicated a flexible network. b) 2DP prepared with amphiphilic monomer 8; STM analysis confirmed local periodicity. Adapted with permission from ref. [66]. c) Terpyridine-based monomer 9 for synthesis of 2D-MOFs; the monolayer sheets resulted free-standing as confirmed by SEM analysis. Adapted with permission from ref. [67]. d) Dipyrrin-based monomer 10 for synthesis of free-standing 2D-MOF monolayers. Adapted with permission from ref. [70]. e) Thiol-based monomer 11 for the synthesis of 2D-MOFs; the monolayers are free-standing according to SEM analysis. Adapted with permission from ref. [72]. $f$ ) Monomers 12a and 12b for the synthesis of 2D-COFs via reversible imine chemistry; local crystallinity was confirmed by SAED analysis, as shown in the diffraction pattern. Adapted with permission from ref. [74]. 


\subsubsection{Pros and Cons of the Air/Water Interface Approach}

The main advantage of the air/water interface over the single crystal approach is that the synthesised polymers are already in monolayer form and as such the amount of monomer utilised is minimal (micrograms). This comes however with a downside as for characterisation only monolayer-sensitive techniques can be used. Spectroscopic methods such as fluorescence, IR, Raman spectroscopy and TERS are relatively straightforward to prove bond formation, however, to prove structural periodicity, non-trivial techniques such as HR-TEM, HR-AFM, STM, GISAXS and SAED are necessary. Moreover, conformational changes in the 2DP structure could be problematic when trying to detect crystallinity, as seen in the example of monomer 7a. ${ }^{[61]}$ As with the single crystal approach, with this method there is also an element of uncertainty and it concerns how the monomer will behave at the interface, namely if it will effectively form monolayers. This last issue, however, can be properly addressed by a careful structural design of the monomer.

\subsection{Other Approaches}

The rising activity in the field of 2DPs has sparked interest in finding alternative synthetic methods apart from the single crystal and the air/water interface approach. While these methods have not yet resulted in true 2DPs meeting the five definition criteria, there is no doubt about their potential for success.

\subsubsection{Covalent Organic Frameworks (COFs)}

The first method intends to access 2DPs from $2 \mathrm{D}$-COFs. $2 \mathrm{D}$-COFs are porous microcrystalline layered materials of organic nature. ${ }^{[75]}$ They are typically synthesised by mixing simple organic building blocks in the right stoichiometry in solution, from which they then precipitate out as microcrystalline powders. The simplicity of this method is clearly attractive, however, order formation and growth reaction happen simultaneously in solution and are not two separated processes as in the single crystal and air/water interface approaches. This inevitably implies formation of defects and requires COFs to be based on dynamic covalent chemistry, ${ }^{[76-78]}$ supposedly imparting susceptibility to the layers towards hydrolysis. So far, despite some progress, single crystals of 2D-COFs are still to be obtained, whereas for 3D-COFs single crystals have been grown. ${ }^{[79,80]}$ Exfoliation of 2D-COFs into single layers is also an open issue. ${ }^{\left[{ }^{[1,82]}\right.}$ and so far only thin sheet stacks could be obtained. In this regard to obtain monolayers, COF chemistry could be applied at the air/water interface, as demonstrated with monomers 12a and $\mathbf{1 2} \mathbf{b}^{[74]}$ and by a similar work of Dai et al. ${ }^{[83]}$ Alternatively, COFs can also be grown on solid substrates, ${ }^{\left[{ }^{84}\right]}$ which brings the topic to the next potential approach towards 2DPs: the synthesis on solid surfaces.

\subsubsection{Synthesis on Solid Surfaces}

In this method monomers are deposited by sublimation or drop-casting on highly flat substrates such as highly oriented pyrolytic graphite (HOPG) or metal surfaces. ${ }^{[85]}$ Typically, irreversible cross-couplings mediated by the metal surface itself are used as connection chemistry, but dynamic covalent chemistry can be also used on HOPG. The main advantage is that structural analysis is straightforward as the substrates onto which the polymerisation occurs can be directly imaged by STM. Unfortunately, there are two main issues that still have to be addressed; the first one concerns the rather small lateral extensions that the polymers (except graphitic ribbons) can achieve: for irreversible bonds they are typically extended over few tens of nanometres, ${ }^{[86,87]}$ while for reversible bond they can reach up to $200 \mathrm{~nm}$. ${ }^{[88,89]}$ The second issue concerns the fact that the obtained polymers cannot be lifted off from the substrates.

\subsubsection{Synthesis in Solution}

The solution approach is in principle very attractive as the monomers could be simply dissolved in a suitable solvent and upon polymerisation sheets would form. However, attempts to synthesise 2DPs via irreversible bond formation in homogeneous solutions are scarce. ${ }^{[90,91]}$ The biggest challenge of this method is ensuring that the growth reaction is exclusively confined in $2 \mathrm{D}$, as there is no pre-organisation of the monomers, nor a templating surface available; out-of-plane additions of the monomers caused by for instance bond rotations on the growing polymer must be prevented. A possible way to circumvent this problem is to create regular supramolecular self-assembled 2D networks in solution, which can be subsequently fixated by covalent bonds. A notable progress in this regard is provided by the work of Yu et al. from the Häner group, in which trifunctional monomers equipped with anthracene-bearing complementary single-stranded DNA chains, self-assemble in solution forming supramolecular hexagonal networks, which are subsequently covalently fixated through [4+4]-cycloaddition of the anthracene moieties $^{[92]}$ (Fig. 7). Periodicity of the network could not be confirmed. For other interesting examples of supramolecular 2D assemblies the reader can refer to the reports of the Zhao group. ${ }^{[93,94]}$

\section{Potential Applications}

2DPs are not only interesting because they expand conceptually the field of polymer chemistry; ${ }^{[95]}$ in fact they can also be envisioned for a variety of applications, which will be discussed in the following paragraphs.

2DPs are periodical intrinsically porous structures, reminiscent of a molecular fisherman's net. Their ultrathin nature (typically 1-2 nm) combined with regularly distributed monodisperse pores make them promising candidates as supported membranes for gas separation. The $1 \mathrm{~nm}$ thickness effectively suppresses diffusion limitation (an advantage over MOF-based membranes ${ }^{[96]}$ ), and a pore diameter of approximately $1 \mathrm{~nm}$ allows to operate in

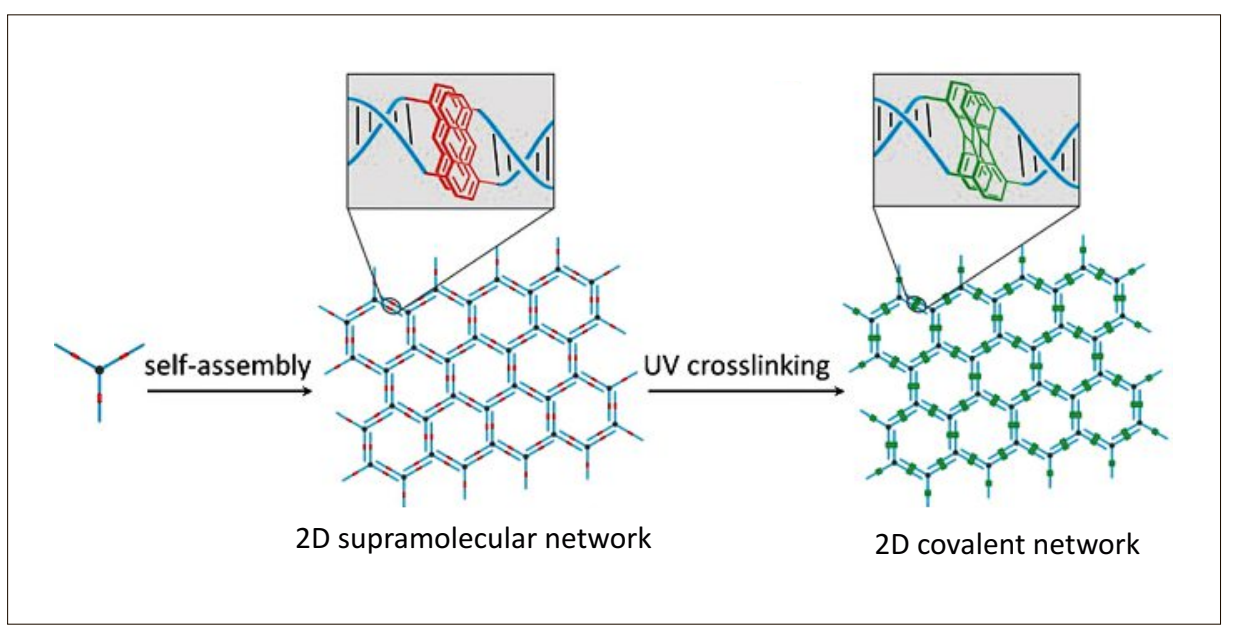

Fig. 7. Supramolecular approach toward 2DPs from Yu et al.: a monomer is equipped with three anthracene-bearing complimentary single stranded DNA chains. In solution, the monomers self-assemble in a regular 2D supramolecular network, forcing the anthracene units in the complimentary strands to stack face-to-face. Photoirradiation dimerises the anthracenes, covalently fixing the network. Adapted with permission from ref. [92]. 
the Knudsen flow regime, where the pore size is equal to or smaller than the mean free path of the gas molecules. With these conditions, the gas molecules will mostly interact with the pores' walls rather than other gas molecules and separation will occur according to the molar mass of the molecules. ${ }^{[97]}$ The pore size depends on the monomer structure but in principle, it could also be tuned by post-polymerisation modifications on the pore rim. This is an advantage compared to graphene-based membranes, where regularly distributed pores are still not accessible. ${ }^{98]}$

In another scenario, the pores could be instead filled by guest molecules, resulting in crystalline composite materials; for applications in photovoltaics for instance, one component could have electron donor character while the other electron acceptor character. In this regard, attempts to co-crystallise monomer $\mathbf{4}$ with fullerenes are currently in progress.

Currently known 2DPs are not conductive, therefore applications as novel dielectrics are being pursued. In particular, their extreme thinness and lateral extension, could be exploited for macroscopically covering entire wafers with an insulating layer, possibly challenging the currently used $\mathrm{BN}_{\mathrm{x}}$.

Another example regards the use of 2DP single crystals as non-linear optics (NLO) media for second harmonic generation, as in the case of monomer 4. Exfoliation of the crystals into thin-sheet packages does not hamper their NLO properties and as such they could be used to modulate optical waveguides in nanosized, tunable coherent light sources. Such devices are currently under investigation.

Regularly distributed functional groups on the surface of a 2DP could be exploited for catalytic purposes as well, if precise post-polymerisation modifications could transform these groups into catalytically active single-sites. This would allow to design catalytic surfaces with a very high population of evenly distributed active sites, with which the study of structure-activity relationships could be simplified. [99]

Finally, for anthracene-based monomers used for the synthesis of 2D covalent monolayers at the air/water interface, the photopolymerisation reaction is typically accompanied by loss of fluorescence due to the dimerisation of the anthracene moieties via [4+4]-cycloaddition. Since dimerisation is thermally reversible, the fluorescence can be in principle recovered, so that the monolayers can be used as rewritable molecular paper. Writing can be performed microscopically with a laser scanning confocal microscope ${ }^{[65]}$ or macroscopically with a handheld laser pointer, and erasing can be performed by thermal treatment. Applications of these monolay- ers in miniaturised optical devices such as meta-lenses are also of great interest.[100]

\section{Summary and Outlook}

The field of 2DPs is still in its infancy, but the interest in 2D materials and the steadily increasing reports in the literature of unambiguously proven structures is pushing more people to join this exciting topic. Much like in the case of linear polymers, the organic chemistry approach for the synthesis of 2DPs offers the possibility to access a wide palette of new functional materials. Application areas of potential societal impact are already being explored, such as the use of 2DP monolayers as ultrathin membranes for gas separation and 2DP sheet stacks in miniaturised devices for NLO.

Challenges are present at all stages, from the monomer design and synthesis, to the polymerisation reaction and all the way to the structural characterisation. This should however not discourage researchers, as once the ice broken, the experience (in terms of success and failures) gathered in the field can only lead to improvement. In fact, apart from the two main established methods for synthesising 2DPs, namely the single crystal and the air/water interface approach, new potential methods are already being developed, among which 2D-COFs and the synthesis in solution through covalent fixation of $2 \mathrm{D}$ supramolecular networks seem promising. Even more creative methods such as molecular weaving are also underway. [101] Improvement in structural analysis of covalent monolayers prepared at the air/water interface is also being pushed forward as SAED has proven successful in detecting crystallinity locally on these sheets. As such, eventually, the covalent monolayer sheets that still await structural elucidation will be addressed. In the meantime, applications for which structural periodicity might not be so crucial are already being explored, such as rewritable molecular paper and ultrathin dielectrics.

The hope is that this article will spark even more interest in this exciting field and will bring scientists from different backgrounds together in order to develop the macromolecular flatland even faster.

\section{Acknowledgements}

The author would like to thank Prof. A. Dieter Schlüter and Prof. Holger Frauenrath for the opportunity to write this article. Many thanks also go to Dr. Payam Payamyar for the valid scientific inputs. Financial support from ETH grants is most gratefully acknowledged.

Received: April 18, 2017
[1] H. Staudinger, Ber. der Dtsch. Chem. Gesell. 1920, 53, 1073 .

[2] The term polymer was actually coined by $\mathbf{J}$ J. Berzelius in 1832 and was used to describe compounds sharing the same empirical formula but different in molecular weight; J.J. Berzelius, 'Årsberättelse om Framstegen i Fysik och Kemi’, Stockholm: P. A. Norsted \& Söner.

[3] P. C. Painter, M. M. Coleman, 'Essentials of Polymer Science and Engineering', DEStech Publications Inc., 2009.

[4] R. Mülhaupt, Angew. Chem. Int. Ed. 2004, 43, 1054.

[5] PlasticsEurope, 'Plastics - the Facts 2016', http://www.plasticseurope.org/Document/ plastics-the-facts-2016-15787.aspx? FolID $=2$

[6] K. S. Novoselov, A. K. Geim, S. V. Morozov, D. Jiang, Y. Zhang, S. V. Dubonos, I. V. Grigorieva, A. A. Firsov, Science 2004, 306, 666.

[7] A. K. Geim, K. S. Novoselov, Nat. Mater 2007, 6, 183.

[8] J. Zhao, H. Liu, Z. Yu, R. Quhe, S. Zhou, Y Wang, C. C. Liu, H. Zhong, N. Han, J. Lu, Y. Yao, K. Wu, Prog. Mater. Sci. 2016, 83, 24.

[9] B. Lalmi, H. Oughaddou, H. Enriquez, A. Kara, Sébastien Vizzini, B. Ealet, B. Aufray, Appl. Phys. Lett. 2010, 97, 2008.

[10] M. E. Dávila, L. Xian, S. Cahangirov, A Rubio, G. Le Lay, New J. Phys. 2014, 16, 1.

[11] F.-F. Zhu, W.-J. Chen, Y. Xu, C.-L. Gao, D.-D Guan, C.-H. Liu, D. Qian, S.-C. Zhang, J.-F Jia, Nat. Mater. 2015, 14, 1020.

[12] S. Saxena, R. P. Chaudhary, S. Shukla, Sci. Rep. 2016, 6, 31073.

[13] H. Liu, A. T. Neal, Z. Zhu, Z. Luo, X. Xu, D. Tománek, P. D. Ye, ACS Nano 2014, 8, 4033.

[14] C. Jin, F. Lin, K. Suenaga, S. Iijima, Phys. Rev. Lett. 2009, 102, 3 .

[15] T. T. Tran, K. Bray, M. J. Ford, M. Toth, I. Aharonovich, Nat. Nanotechnol. 2015, 11, 1.

[16] P. Miró, M. Audiffred, T. Heine, Chem. Soc. Rev. 2014, 43, 6537.

[17] A. Gupta, T. Sakthivel, S. Seal, Prog. Mater Sci. 2015, 73, 44.

[18] R. Mas-Ballesté, C. Gómez-Navarro, J. GómezHerrero, F. Zamora, Nanoscale 2011, 3, 20.

[19] K. S. Novoselov, D. Jiang, F. Schedin, T. J. Booth, V. V Khotkevich, S. V Morozov, A. K. Geim, Proc. Natl. Acad. Sci. U. S. A. $\mathbf{2 0 0 5}$ 102, 10451.

[20] G. Liu, N. Komatsu, ChemPhysChem 2016 , $17,1557$.

[21] A. Reina, X. Jia, J. Ho, D. Nezich, H. Son, V. Bulovic, M. S. Dresselhaus, J. Kong, Nano Lett. 2009, 9, 30.

[22] Y. H. Lee, X. Q. Zhang, W. Zhang, M. T. Chang, C. Te Lin, K. Di Chang, Y. C. Yu, J. T. W. Wang, C. S. Chang, L. J. Li, T. W. Lin, $A d v$. Mater. 2012, 24, 2320.

[23] J. Yu, J. Li, W. Zhang, H. Chang, Chem. Sci. 2015, 6, 6705 .

[24] X. Li, H. Zhu, J. Mater. 2015, 1, 33.

[25] S. J. Kim, K. Choi, B. Lee, Y. Kim, B. H. Hong, Annu. Rev. Mater. Res. 2015, 45, 63.

[26] J. M. Englert, C. Dotzer, G. Yang, M. Schmid C. Papp, J. M. Gottfried, H.-P. Steinrück, E. Spiecker, F. Hauke, A. Hirsch, Nat. Chem. 2011, 3, 279.

[27] T. Kuila, S. Bose, A. K. Mishra, P. Khanra, N H. Kim, J. H. Lee, Prog. Mater. Sci. 2012, 57 , 1061

[28] K. C. Kemp, V. Georgakilas, M. Otyepka, A. B Bourlinos, V. Chandra, N. Kim, K. C. Kemp, P. Hobza, R. Zboril, K. S. Kim, Chem. Rev. 2012 $112,6156$.

[29] Q. Weng, X. Wang, X. Wang, Y. Bando, D Golberg, Chem. Soc. Rev. 2016, 45, 3989.

[30] Y. Sugiyama, H. Okamoto, T. Mitsuoka, T Morikawa, K. Nakanishi, T. Ohta, H. Nakano, J. Am. Chem. Soc. 2010, 132, 5946. 
[31] G. Gee, E. K. Rideal, Proc. R. Soc. A 1935 , $153,116$.

[32] G. Gee, Proc. R. Soc. A 1935, 153, 129.

[33] T. Kunitake, Angew. Chem. Int. Ed. Engl. 1992, 31,709 .

[34] S. I. Stupp, S. Son, L. S. Li, H. C. Lin, M. Keser, J. Am. Chem. Soc. 1995, 117, 5212.

[35] J. Michl, T. F. Magnera, Proc. Natl. Acad. Sci. 2002, 99, 4788.

[36] J. Sakamoto, J. Van Heijst, O. Lukin, A. D. Schlüter, Angew. Chem. Int. Ed. 2009, 48, 1030.

[37] P. Kissel, R. Erni, W. B. Schweizer, M. D. Rossell, B. T. King, T. Bauer, S. Götzinger, A. D. Schlüter, J. Sakamoto, Nat. Chem. 2012, 4, 287.

[38] J. W. Colson, W. R. Dichtel, Nat. Chem. 2013 $5,453$.

[39] Y. Zang, T. Aoki, M. Teraguchi, T. Kaneko, L. Ma, H. Jia, Polym. Rev. 2015, 55, 57.

[40] D. Rodríguez-San-Miguel, P. Amo-Ochoa, F. Zamora, Chem. Commun. 2016, 52, 4113.

[41] B. M. D. Cohen, G. M. J. Schmidt, J. Chem Soc. 1964, 1996.

[42] G. M. J. Schmidt, Pure Appl. Chem. 1971, 27 , 647.

[43] G. Wegner, Z. Naturforsch. 1969, 24 B, 824.

[44] M. Hasegawa, Y. Suzuki, J. Polym. Sci. Part B Polym. Lett. 1967, 5, 813.

[45] R. B. Woodward, R. Hoffmann, Angew. Chem. Int. Ed. Engl. 1969, 8, 781.

[46] R. Bhola, P. Payamyar, D. J. Murray, B. Kumar, A. J. Teator, M. U. Schmidt, S. M. Hammer, A. Saha, J. Sakamoto, A. D. Schlüter, B. T. King, J. Am. Chem. Soc. 2013, 135, 14134

[47] P. Kissel, D. J. Murray, W. J. Wulftange, V. J. Catalano, B. T. King, Nat. Chem. 2014, 6, 774

[48] M. J. Kory, M. Wörle, T. Weber, P. Payamyar, S. W. van de Poll, J. Dshemuchadse, N. Trapp, A. D. Schlüter, Nat. Chem. 2014, 6, 779 .

[49] R. Z. Lange, G. Hofer, T. Weber, A. D. Schlüter, J. Am. Chem. Soc. 2017, 139, 2053.

[50] K. Novak, V. Enkelmann, G. Wegner, K. B. Wagener, Angew. Chem. Int. Ed. Engl. 1993, 32, 1614.

[51] V. Enkelmann, Mol. Cryst. Liq. Cryst. Sci. Technol. 1998, 313, 15.

[52] I. Halasz, Cryst. Growth Des. 2010, 10, 2817.

[53] G. R. Desiraju, J. Am. Chem. Soc. 2013, 135, 9952.

[54] M. A. Neumann, F. J. J. Leusen, J. Kendrick Angew. Chem. Int. Ed. 2008, 47, 2427.

[55] G. M. Day, T. G. Cooper, A. J. Cruz-Cabeza, K E. Hejczyk, H. L. Ammon, S. X. M. Boerrigter, J. S. Tan, R. G. Della Valle, E. Venuti, J. Jose, S. R. Gadre, G. R. Desiraju, T. S. Thakur, B. P. Van Eijck, J. C. Facelli, V. E. Bazterra, M. B. Ferraro, D. W. M. Hofmann, M. A. Neumann, F. J. J. Leusen, J. Kendrick, S. L. Price, A J. Misquitta, P. G. Karamertzanis, G. W. A. Welch, H. A. Scheraga, Y. A. Arnautova, M U. Schmidt, J. Van De Streek, A. K. Wolf, B. Schweizer, Acta Crystallogr. Sect. B Struct. Sci. 2009, 65, 107.

[56] M. Servalli, N. Trapp, M. Wörle, F.-G. Klärner, J. Org. Chem. 2016, 81
[57] M. Servalli, N. Trapp, M. Solar, A. D. Schlüter, Cryst. Growth Des. 2017, doi:10.1021/acs. cgd.7b00367, accepted.

[58] I. Langmuir, J. Am. Chem. Soc. 1917, 39, 1848.

[59] I. Langmuir, Trans. Faraday Soc. 1920, 15, 62

[60] A. Braslau, M. Deutsch, P. S. Pershan, A. H Weiss, J. Als-Nielsen, J. Bohr, Phys. Rev. Lett. 1985, 54, 114.

[61] P. Payamyar, K. Kaja, C. Ruiz-Vargas, A. Stemmer, D. J. Murray, C. J. Johnson, B. T. King, F. Schiffmann, J. Vandevondele, A Renn, S. Götzinger, P. Ceroni, A. Schütz, L. T. Lee, Z. Zheng, J. Sakamoto, A. D. Schlüter, Adv. Mater. 2014, 26, 2052

62] L. Opilik, P. Payamyar, J. Szczerbinsk, A. P. Schutz, M. Servalli, T. Hungerland, A. D. Schlüter, R. Zenobi, ACS Nano 2015, 9, 4252.

63] Y. Chen, M. Li, P. Payamyar, Z. Zheng, J. Sakamoto, A. D. Schlüter, ACS Macro Lett. 2014, 3, 153 .

[64] P. Payamyar, M. Servalli, T. Hungerland, A P. Schütz, Z. Zheng, A. Borgschulte, A. D Schlüter, Macromol. Rapid Commun. 2015, $36,151$.

[65] V. Müller, T. Hungerland, M. Baljozovic, T. Jung, N. D. Spencer, H. Eghlidi, P. Payamyar, A. D. Schlüter, Adv. Mater. 2017, accepted.

[66] D. J. Murray, D. D. Patterson, P. Payamyar, R. Bhola, W. Song, M. Lackinger, A. D. Schlüter, B. T. King, J. Am. Chem. Soc. 2015, 137, 3450.

[67] T. Bauer, Z. Zheng, A. Renn, R. Enning, A Stemmer, J. Sakamoto, A. D. Schlüter, Angew. Chem. Int. Ed. 2011, 50, 7879.

[68] Z. Zheng, C. S. Ruiz-vargas, T. Bauer, A Rossi, P. Payamyar, A. Schütz, A. Stemmer, J. Sakamoto, A. D. Schlüter, Macromol. Rapid Commun. 2013, 34, 1670 .

[69] R. Sakamoto, T. Iwashima, M. Tsuchiya, R. Toyoda, R. Matsuoka, J. F. Kögel, S Kusaka, K. Hoshiko, T. Yagi, T. Nagayama, H Nishihara, J. Mater. Chem. A 2015, 3, 15357.

[70] R. Sakamoto, K. Hoshiko, Q. Liu, T. Yagi, T. Nagayama, S. Kusaka, M. Tsuchiya, Y. Kitagawa, W.-Y. Wong, H. Nishihara, Nat. Commun. 2015, 6, 6713.

[71] T. Kambe, R. Sakamoto, K. Hoshiko, K. Takada, M. Miyachi, J. Ryu, S. Sasaki, J. Kim, K. Nakazato, M. Takata, H. Nishihara, J. Am Chem. Soc. 2013, 135, 2462.

[72] R. Dong, M. Pfeffermann, H. Liang, Z. Zheng, X. Zhu, J. Zhang, X. Feng, Angew. Chem. Int Ed. 2015, 54, 12058.

[73] R. Makiura, O. Konovalov, Sci. Rep. 2013, 3, 1.

[74] H. Sahabudeen, H. Qi, B. A. Glatz, D. Tranca, R. Dong, Y. Hou, T. Zhang, C. Kuttner, T. Lehnert, G. Seifert, U. Kaiser, A. Fery, Z Zheng, X. Feng, Nat. Commun. 2016, 7, 13461.

[75] N. W. Ockwig, A. P. Cote, M. O. Keeffe, A. J. Matzger, O. M. Yaghi, Science 2005, 310, 1166.

[76] J.-M. Lehn, Chem. Eur. J. 1999, 5, 2455.

[77] S. J. Rowan, S. J. Cantrill, G. R. L. Cousins, J. K. M. Sanders, J. F. Stoddart, Angew. Chem. Int. Ed. 2002, 41, 898.
[78] Y. Jin, C. Yu, R. J. Denman, W. Zhang, Chem. Soc. Rev. 2013, 42, 6634

[79] Y. B. Zhang, J. Su, H. Furukawa, Y. Yun, F. Gándara, A. Duong, X. Zou, O. M. Yaghi, J. Am. Chem. Soc. 2013, 135, 16336.

[80] D. Beaudoin, T. Maris, J. D. Wuest, Nat. Chem. 2013, 5, 830 .

[81] I. Berlanga, M. L. Ruiz-González, J. M. González-Calbet, J. L. G. Fierro, R. MasBallesté, F. Zamora, Small 2011, 7, 1207.

[82] D. N. Bunck, W. R. Dichtel, J. Am. Chem. Soc. 2013, 135, 14952 .

[83] W. Dai, F. Shao, J. Szczerbiński, R. McCaffrey, R. Zenobi, Y. Jin, A. D. Schlüter, W. Zhang, Angew. Chem. Int. Ed. 2016, 55, 213.

[84] J. W. Colson, A. R. Woll, A. Mukherjee, M. P. Levendorf, E. L. Spitler, V. B. Shields, M. G. Spencer, J. Park, W. R. Dichtel, Science 2011, 332, 228.

[85] M. Lackinger, Polym. Int. 2015, 64, 1073.

[86] M. Lackinger, W. M. Heckl, J. Phys. D. Appl. Phys. 2011, 44, 464011.

[87] Q. Fan, J. M. Gottfried, J. Zhu, Acc. Chem. Res. 2015, 48, 2484

[88] Y. Yu, J.-B. Lin, Y. Wang, Q. Zeng, S. Lei, Chem. Commun. 2016, 52, 6609.

[89] C.-Z. Guan, D. Wang, L.-J. Wan, Chem. Commun. 2012, 48, 2943.

[90] K. Baek, G. Yun, Y. Kim, D. Kim, R. Hota, I. Hwang, D. Xu, Y. H. Ko, G. H. Gu, J. H. Suh, C. G. Park, B. J. Sung, K. Kim, J. Am. Chem. Soc. 2013, 135, 6523.

[91] T. Zhou, F. Lin, Z. Li, X. Zhao, Macromolecules 2013, 46, 7745

[92] H. Yu, D. T. L. Alexander, U. Aschauer, R. Häner, Angew. Chem. Int. Ed. 2017, 57, 5040.

[93] T.-Y. Zhou, Q.-Y. Qi, Q.-L. Zhao, J. Fu, Y. Liu, Z. Ma, X. Zhao, Polym. Chem. 2015, 6, 3018

[94] K. Da Zhang, J. Tian, D. Hanifi, Y. Zhang, A C. H. Sue, T. Y. Zhou, L. Zhang, X. Zhao, Y. Liu, Z. T. Li, J. Am. Chem. Soc. 2013, 135, 17913.

[95] A. D. Schlüter, P. Payamyar, H. C. Öttinger, Macromol. Rapid Commun. 2016, 37, 1638.

[96] T. Rodenas, I. Luz, G. Prieto, B. Seoane, H Miro, A. Corma, F. Kapteijn, F. X. Llabrés Xamena, J. Gascon, Nat. Mater. 2015, 14, 48.

[97] M. Vargas, S. Naris, D. Valougeorgis, S. Pantazis, K. Jousten, Vacuum 2014, 109, 385

[98] K. Celebi, J. Buchheim, R. M. Wyss, A Droudian, P. Gasser, I. Shorubalko, J.-I. J.-I. Kye, C. Lee, H. G. Park, Science 2014, 344, 289

[99] J. D. A. Pelletier, J.-M. Basset, Acc. Chem. Res. 2016, 49, 664 .

[100] M. Khorasaninejad, W. T. Chen, R. C. Devlin, J. Oh, a. Y. Zhu, F. Capasso, Science 2016, 352, 1190.

[101] Z. Wang, A. Błaszczyk, O. Fuhr, S. Heissler, C. Wöll, M. Mayor, Nat. Commun. 2017, 8, 14442. 